\title{
ON THE SOLVABILITY OF FINITE GROUPS AND THE NUMBER OF SYLOW 2-SUBGROUPS
}

\author{
RUSONG YANG, KAIRAN YANG AND RULIN SHEN*
}

\begin{abstract}
Let $G$ be a finite group. Denoted by $n_{2}(G)$ the number of Sylow 2-subgroups of $G$. In this paper, we prove if $G$ is non-solvable and $n_{2}(G)$ is a power of a prime $p$, then $p$ is a Fermat prime.
\end{abstract}

\section{INTRODUCTION}

Let $G$ be a finite group and $p$ a prime. We denote by $n_{p}(G)$ the number of Sylow $p$-subgroups of $G$, which is called Sylow $p$-number of $G$ (hereinafter referred to as Sylow number). The influence of the number of Sylow subgroups in finite groups on group structure is a very meaningful research topic. In 1967, M. Hall [1], studied the number of Sylow subgroups in finite groups, and proved that solvable group have solvable Sylow numbers, and 22 is never a Sylow 3-number and 21 a Sylow 5-number. In 1995, Zhang [2], proved that a finite group $G$ is $p$-nilpotent if and only if $p$ is prime to every sylow number of G. In 2003, G. Navarro [3] proved that if $G$ is $p$-solvable, then $n_{p}(H)$ divides $n_{p}(G)$ for every $H \leq G$. In 2016 [4], Li and Liu classified finite non-abelian simple group with only solvable Sylow numbers. We say that a group $G$ satisfies $\operatorname{DivSyl}(p)$ if $n_{p}(H)$ divides $n_{p}(G)$ for every $H \leq G$. In 2018, Guo and E. P. Vdovin [5] generalized the results of G. Navarro, and proved that G satisfies $\operatorname{Div} \operatorname{Syl}(p)$ provided every non-abelian composition factor of $G$ satisfies $\operatorname{Div} \operatorname{Syl}(p)$. Recently, Wu [6] proved that finite simple group does not satisfy $\operatorname{Div} \operatorname{Syl}(p)$. In this paper, we will study the relationship between the number of Sylow 2-subgroups and the solvability of groups. Obviously, the number of Sylow 2-subgroups is odd. By the famous Feit-Thompson odd order Theorem, if the number of Sylow 2 -subgroups of $G$ is 1 , then $G$ is solvable. A natural question is whether can we determine the solvability of $G$ if the number of Sylow 2-subgroups of $G$ is given? In this paper, we study the case that Sylow 2-numbers is a prime power and obtain the following main result.

Theorem. If $G$ is non-solvable and the number of Sylow 2-subgroups of $G$ is a power of a prime $p$, then $p$ is a Fermat prime.

Note that a Fermat prime above means a prime of the type $2^{a}+1$. Also when $n_{2}(G)=3, G$ is solvable. If $k \geq 2$, then there exists a non-solvable group $G=P S L(2,8) \times S_{3}^{k-2}$ such that

Department of Mathematics, Hubei Minzu University, Enshi 445000, Hubei, P.R. China

*CORRESPONDING AUTHOR

E-mail addresses: email1, email2, rshen@hbmy.edu.cn.

Key words and phrases. Sylow 2-numbers; non-solvable groups; Fermat prime.

Project supported by the NSF of China (Grant No. 12161035).

Received 12/11/2021. 
$n_{2}(G)=3^{k}$. Suppose that $p=2^{a}+1>3$ is a Fermat prime, then there exists a non-solvable group $G=P S L\left(2,2^{a}\right)^{k}$ such that $n_{2}(G)=p^{k}$.

\section{Some Lemmas}

Lemma 2.1 Let $G$ be a finite group and $N$ a normal subgroup of $G$, then both $n_{2}(N)$ and $n_{2}(G / N)$ divide $n_{2}(G)$.

Proof. Let $P_{2}$ be a Sylow 2-subgroup of $G$. By Theorem 2.1 in [1], we have $n_{2}(G)=a_{2} b_{2} c_{2}$, where $a_{2}$ is the number of Sylow 2-subgroups in $G / N, b_{2}$ is the number of Sylow 2-subgroups in $N$ and $c_{2}$ is the number of Sylow 2-subgroups in $N_{P_{2} N}\left(P_{2} \cap N\right) / P_{2} \cap N$. Thus we get both $n_{2}(N)$ and $n_{2}(G / N)$ divide $n_{2}(G)$, as required.

Lemma 2.2 Let $P_{2}$ be a Sylow 2-group of $P S L(2, q)$, where $q$ is power of odd prime, then

(1) if $3<q \equiv \pm 3(\bmod 8)$, then $N_{P S L(2, q)}\left(P_{2}\right) \cong A_{4}$,

(2) if $3<q \equiv \pm 1(\bmod 8)$, then $N_{P S L(2, q)}\left(P_{2}\right) \cong P_{2}$.

Proof. Let $G$ be a finite non-abelian simple group and $P_{2}$ a Sylow 2-subgroup of $G$. By Corollary in [7], we get that $N_{G}\left(P_{2}\right) \cong P_{2}$, except in the following case: $G \cong P S L(2, q)$, where $3<q \equiv \pm 3(\bmod 8)$ and $N_{G}\left(P_{2}\right) \cong A_{4}$. Therefore, for $3<q \equiv \pm 3(\bmod 8)$, we have $N_{P S L(2, q)}\left(P_{2}\right) \cong A_{4}$. For $3<q \equiv \pm 1(\bmod 8)$, we know that $N_{P S L(2, q)}\left(P_{2}\right) \cong P_{2}$, as required.

The following Lemma gives the formula for calculating the number of Sylow 2-subgroups of $\operatorname{PSL}(2, q)$. We denoted by $2^{\prime}$ and $n_{2^{\prime}}$ the set of all odd primes and the $2^{\prime}$-part of $n$ (i.e. the largest odd factor of $n$ ), respectively.

\section{Lemma 2.3}

$$
n_{2}(P S L(2, q))=\left\{\begin{array}{l}
q+1, \text { where } q=2^{f} \text { for } f \text { is a positive integer } \\
\frac{q\left(q^{2}-1\right)}{24}, 3<q \equiv \pm 3(\bmod 8) \\
\left(\frac{q\left(q^{2}-1\right)}{2}\right)_{2^{\prime}}, 3<q \equiv \pm 1(\bmod 8) .
\end{array}\right.
$$

Proof. First we denote by $P_{2}$ and $n_{2}$ the Sylow 2-subgroups of $P S L(2, q)$ and the number of Sylow 2-subgroups of $\operatorname{PSL}(2, q)$, respectively. Next we get, by the second Sylow theorem in [8], that $n_{2}=\left|G: N_{G}\left(P_{2}\right)\right|$. If $q=2^{f}$, then $\left|N_{P S L(2, q)}\left(P_{2}\right)\right|=q(q-1)$ by [9], and so $n_{2}=\left|P S L(2, q): N_{P S L(2, q)}\left(P_{2}\right)\right|=\frac{q\left(q^{2}-1\right)}{q(q-1)}=q+1$. If $3<q \equiv \pm 3(\bmod 8)$, by Lemma 1.2 , we have $N_{P S L(2, q)}\left(P_{2}\right) \cong A_{4}$, and then $n_{2}=\left|P S L(2, q): A_{4}\right|=\frac{q\left(q^{2}-1\right)}{24}$. Also by Lemma 1.2, if $3<q \equiv \pm 1(\bmod 8)$, then $N_{P S L(2, q)}\left(P_{2}\right) \cong P_{2}$, and so $n_{2}=\left|P S L(2, q): P_{2}\right|=\left(\frac{q\left(q^{2}-1\right)}{2}\right)_{2^{\prime}}$, as required.

Lemma 2.4 Let $p$ and $r$ be primes, and $m$ and $n$ be positive integers. Then there exists a prime $s$ such that $s \mid p^{n}-1$ and $s \nmid p^{m}-1$, where $m<n$, except $(p, n)=(2,6)$ or $p=2^{r}-1$ is a Mersenne prime and $n=2$.

Proof. The Lemma follows from [10] and [11]. 
Note that the above $s$ is called the n-th primitive prime factors of $p$, also known as the Zsigmondy primes. The following Lemma gives a complete classification of simple groups whose index of maximal subgroups are prime powers.

Lemma 2.5 Let $G$ be a finite non-abelian simple group with $H<G$ and $|G: H|=p^{n}$, p prime. One of the following holds.

(1) $G=A_{m}$ and $H \cong A_{m-1}$ with $m=p^{n}$,

(2) $G=P S L(m, q)$ and $H$ is the stabilizer of a line or hyperplane. Then $|G: H|=\frac{q^{m}-1}{q-1}=p^{n}$ (Note $m$ must be prime),

(3) $G=P S L(2,11)$ and $H \cong A_{5}$,

(4) $G=M_{23}$ and $H \cong M_{22}$ or $G=M_{11}$ and $H \cong M_{10}$,

(5) $G=P S U(4,2) \cong P S p(4,3)$ and $H$ is the parabolic subgroup of index 27 .

Proof. The Lemma follows immediately from Theorem 1 in [12].

Lemma 2.6 Let $G$ be a finite non-abelian simple group and $P_{2}$ a Sylow 2-group of $G$. If $\left|G: N_{G}\left(P_{2}\right)\right|$ is a prime power, then $G \cong P S L(2, q)$.

Proof. Let $H$ be a maximal subgroup of $G$. Suppose that $\left|G: N_{G}\left(P_{2}\right)\right|$ is a power of a prime $p$, then we set $\left|G: N_{G}\left(P_{2}\right)\right|=p^{k}$, where $k$ is a positive integer. Now $|G: H|$ is also a prime power since $N_{G}\left(P_{2}\right) \leq H$. Furthermore, by Lemma 1.5, we get that $G$ is isomorphic to one of the following groups: $A_{m}$ with $m=p^{n}$ and $k \geq n, P S L(m, q)$ for $m$ prime, $\operatorname{PSL}(2,11), M_{23}$, $M_{11}, P S U(4,2)$.

If $G \cong A_{m}$ with $m=p^{n}$, then $|G|=\frac{m !}{2}$. By Corollary in [7], we know that $N_{G}\left(P_{2}\right)=P_{2}$, thus $n_{2}\left(A_{m}\right)=\left|A_{m}: N_{A_{m}}\left(P_{2}\right)\right|=\left(\frac{p^{n} \cdot\left(p^{n}-1\right) \cdot\left(p^{n}-2\right) \cdots \cdot 2 \cdot 1}{2}\right)_{2^{\prime}}$, which contradicts $n_{2}\left(A_{m}\right)=p^{k}$ since $p^{n} \geq 5$.

If $G \cong P S L(m, q)$ for $m$ prime, then $|G|=\frac{1}{(m, q-1)} q^{\frac{m(m-1)}{2}} \prod_{i=1}^{m-1}\left(q^{i+1}-1\right)$. Suppose first that the characteristic of $G$ is 2 and $m \geq 3$, we see that $N_{G}\left(P_{2}\right)$ is a Borel subgroup $B$ of $G$ which differs from $P_{2}$ by Corollary in [7]. Moreover, by [13], we get that $B$ is the subgroup of all lower-triangular matrices, and then $B \cong P_{2}: D$, where $D$ of $P S L(m, q)$ consisting of all diagonal matrices is easily seen to be a subgroup of order $\frac{(q-1)^{m-1}}{(m, q-1)}$. Hence

$$
\begin{aligned}
n_{2}(P S L(m, q)) & =\left|P S L(m, q): N_{P S L(m, q)}\left(P_{2}\right)\right| \\
& =|P S L(m, q): B|=\frac{q^{\frac{m(m-1)}{2}} \cdot \prod_{i=1}^{m-1}\left(q^{i+1}-1\right) \cdot(m, q-1)}{(m, q-1) \cdot(q-1)^{m-1} \cdot\left|P_{2}\right|} \\
& =\left(\frac{q^{\frac{m(m-1)}{2}} \cdot \prod_{i=1}^{m-1}\left(q^{i+1}-1\right)}{(q-1)^{m-1}}\right)_{2^{\prime}}=\frac{\left(q^{2}-1\right)\left(q^{3}-1\right) \cdots\left(q^{m}-1\right)}{(q-1)^{m-1}} .
\end{aligned}
$$

By Lemma 2.4 the existence of primitive prime factor, there must exists 2-th and 3-th primitive prime factor of $q$ in $n_{2}(P S L(m, q))$, so $n_{2}(P S L(m, q))$ has at least two different prime factors, and then $n_{2}(P S L(m, q))=p^{k}$ is impossible. Next suppose that $q$ is odd and $m \geq 3$. By Corollary in [7], we get that $P_{2} \neq N_{G}\left(P_{2}\right)=P_{2} \times C_{1} \times \cdots \times C_{t-1}$, where the number $t \geq$ 2 can be found from the 2-adic expansion $m=2^{s_{1}}+\cdots+2^{s_{t}}, s_{1}>\cdots>s_{t} \geq 0$, and $C_{1}, \cdots, C_{t-2}, C_{t-1}$ are cyclic groups of orders $(q+1)_{2^{\prime}}, \cdots,(q+1)_{2^{\prime}}, \frac{(q+1)_{2^{\prime}}}{(q+1, m)_{2^{\prime}}}$, respectively. Thus $\left|N_{G}\left(P_{2}\right)\right|=\frac{\left|P_{2}\right| \cdot\left((q+1)_{2^{\prime}}\right)^{t-1}}{(q+1, m)_{2^{\prime}}}$, and then $n_{2}(P S L(m, q))=\left|P S L(m, q): N_{P S L(m, q)}\left(P_{2}\right)\right|=$ $\left(\frac{q^{\frac{m(m-1)}{2}} \cdot \prod_{i=1}^{m-1}\left(q^{i+1}-1\right) \cdot(q+1, m)_{2^{\prime}}}{(m, q-1) \cdot\left((q+1)_{2^{\prime}}\right)^{t-1}}\right)_{2^{\prime}}$. We set $i+1=n$. For $n \neq 2$ or $q$ is not a Mersenne prime, we know that $n_{2}(P S L(m, q))$ has at least two different primitive prime factors by Lemma 1.4 
the existence of primitive prime factor, and so $n_{2}(P S L(m, q))=p^{k}$ is impossible. Next we consider the case $n=2$ or $q$ is a Mersenne prime of the type $2^{r}-1$. Since $m \geq 3$ is a prime, we conclude that $n_{2}(P S L(m, q))=\left|P S L(m, q): N_{P S L(m, q)}\left(P_{2}\right)\right|=\left(\frac{q^{\frac{m(m-1)}{2}} \cdot \prod_{i=1}^{m-1}\left(q^{i+1}-1\right)}{(m, q-1)}\right)_{2^{\prime}}$ and $q^{n}-1=\left(2^{r}-1\right)^{2}-1=2^{r+1}\left(2^{r-1}-1\right)$. Assume first that $r \neq 7$, we see, by Lemma 1.4, that $n_{2}(P S L(m, q))$ has at least two different prime factors, and so $n_{2}(P S L(m, q))=p^{k}$ is impossible. Next assume that $r=7$. If $m=3$, then $n_{2}(P S L(3,127))=\left(\frac{127^{3} \cdot\left(127^{2}-1\right) \cdot\left(127^{3}-1\right)}{3}\right)_{2^{\prime}}=$ $\left(2^{9} \cdot 3^{5} \cdot 7^{2} \cdot 127^{3} \cdot 5419\right)_{2^{\prime}}=3^{5} \cdot 7^{2} \cdot 127^{3} \cdot 5419$, which contradicts $n_{2}(P S L(m, q))=p^{k}$. If $m \geq 5$ then, by Lemma 1.4 the existence of primitive prime factor, there must exists 3 -th, 4 -th and 5-th primitive prime factor of $q$ in $n_{2}(P S L(m, q))$, and so $n_{2}(P S L(m, q))$ has at least three different prime factors, contrary to $n_{2}(P S L(m, q))=p^{k}$. From the above, we get $m=2$, and then $G \cong P S L(2, q)$.

If $G \cong M_{23}$, then $|G|=2^{7} \cdot 3^{2} \cdot 5 \cdot 7 \cdot 11 \cdot 23$. Moreover, by Corollary in [7], we see that $N_{M_{23}}\left(P_{2}\right)=P_{2}$, thus $n_{2}\left(M_{23}\right)=\left|M_{23}: N_{M_{23}}\left(P_{2}\right)\right|=\left|M_{23}: P_{2}\right|=3^{2} \cdot 5 \cdot 7 \cdot 11 \cdot 23$, which contradicts the fact that $n_{2}\left(M_{23}\right)=p^{k}$.

If $G \cong M_{11}$, then $|G|=2^{4} \cdot 3^{2} \cdot 5 \cdot 11$. Also by Corollary in [7], we have $N_{M_{11}}\left(P_{2}\right)=P_{2}$, hence $n_{2}\left(M_{11}\right)=\left|M_{11}: N_{M_{11}}\left(P_{2}\right)\right|=\left|M_{11}: P_{2}\right|=3^{2} \cdot 5 \cdot 11$, contrary to $n_{2}\left(M_{11}\right)=p^{k}$.

If $G \cong P S U(4,2)$, then $|P S U(4,2)|=25920$. On the other hand, by the GAP [14] SmallGroups package, we get $N_{G}\left(P_{2}\right)=192$, so

$$
n_{2}(P S U(4,2))=\left|P S U(4,2): N_{P S U(4,2)}\left(P_{2}\right)\right|=135=3^{3} \cdot 5,
$$

which contradicts $n_{2}(P S U(4,2))=p^{k}$, as required.

Lemma 2.7 If $n_{2}(P S L(2, q))=p^{k}$, where $p$ is a prime and $k$ a positive integer, then $p$ is Fermat.

Proof. We set $n_{2}(P S L(2, q))=p^{k}$. By Lemma 1.3, we divide three cases.

Case $I$. If $n_{2}(P S L(2, q))=q+1$, where $q=2^{f}$ for $f$ is a positive integer, then we have $2^{f}+1=p^{k}$, and so $p^{k}-1=2^{f}$. Furthermore, we conclude that $p-1 \mid 2^{f}$, then $p-1=2^{f^{\prime}}$, where $f^{\prime} \leq f$ is a positive integer. Thus $p=2^{f^{\prime}}+1$ is Fermat.

Case $I I$. If $n_{2}(P S L(2, q))=\frac{q\left(q^{2}-1\right)}{24}$, where $3<q \equiv \pm 3(\bmod 8)$, then $\frac{q\left(q^{2}-1\right)}{24}=p^{k}$, and so $\frac{q^{2}-1}{24}=1$. Furthermore we get $q=p=5$ is a Fermat prime.

Case $I I I$. If $n_{2}(P S L(2, q))=\left(\frac{q\left(q^{2}-1\right)}{2}\right)_{2^{\prime}}$, where $3<q \equiv \pm 1(\bmod 8)$, then $\left(\frac{q\left(q^{2}-1\right)}{2}\right)_{2^{\prime}}=p^{k}$, and so $\left(q^{2}-1\right)_{2^{\prime}}=1$. Furthermore we set $q^{2}-1=2^{l}$, where $l$ is a positive integer. Since $q>3$, we have $q^{2}-1 \equiv 0(\bmod 3)$, contrary to $q^{2}-1=2^{l}$.

Therefore, if $n_{2}(\operatorname{PSL}(2, q))=p^{k}$, then $p$ is a Fermat prime, as required.

\section{Proof of Main Result}

By Lemma 2.1, the Sylow number of the normal subgroups and quotient group of $G$ is still a power of a prime $p$. So we need prove if $p$ is not a Fermat prime and $n_{2}(G)$ is a power of a prime $p$, then $G$ is solvable. Let $\mathrm{G}$ be a counterexample of a minimal order non-solvable group satisfying $n_{2}(G)=p^{k}$ and $p$ is not a Fermat prime, and $k$ is a positive integer. By Lemma 1.1, if the number of Sylow 2-subgroups of normal subgroup and factor subgroup of $\mathrm{G}$ is a power of $p$, then $G$ must be a non-abelian simple group. Furthermore by lemma 1.6, $G \cong P S L(2, q)$. And by Lemma 1.7, we get $p$ is a Fermat prime, a contradiction. As required. 


\section{REFERENCES}

[1] M. Hall, On the number of Sylow subgroups in a finite group, J. Algebra, 7 (3) (1967) 363-371.

[2] J.P. Zhang, Sylow numbers of finite groups, J. Algebra, 176 (1) (1995) 111-123. https://doi.org/10. 1006/jabr.1995.1235.

[3] G. Navarro, Number of Sylow subgroups in p-solvable groups, Proc. Amer. Math. Soc. 131 (10) (2003) 3019-3021. https://doi.org/10.1090/S0002-9939-03-06884-9.

[4] T.-Z. Li, Y.-J. Liu, Mersenne primes and solvable Sylow numbers, J. Algebra Appl. 15 (9) (2016) 1650163. https://doi.org/10.1142/S0219498816501632.

[5] W. Guo, E.P. Vdovin, Number of Sylow subgroups in finite groups, Journal of Group Theory. 21 (4) (2018) 695?712. https://doi.org/10.1515/jgth-2018-0010.

[6] Z. Wu, On the number of Sylow subgroups in finite simple groups, J. Algebra Appl. 20 (2021) 2150115. https://doi.org/10.1142/S0219498821501152.

[7] A.S. Kondrat?ev, Normalizers of the Sylow 2-subgroups in finite simple groups, Math. Notes. 78 (2005) 338?346. https://doi.org/10.1007/s11006-005-0133-9.

[8] M. Hall, The theory of groups, Macmillan, 1959.

[9] C.M. Chen, Internal and external- $\sum$ groups and minimal non- $\sum$ groups, Southwest Normal University Press, 1988.

[10] R.L. Shen, J.T. Shi, C.G. Shao, et al. A note on number of Sylow subgroups of finite groups, J. ShangHai Univ. 016 (006) (2010) 639-642.

[11] K. Zsigmondy, Zur theorie der potenzreste, Monatsh Math. und Phys. B3 (1892) 265-284.

[12] R.M. Guralnick, Subgroups of prime power index in a simple group, J. Algebra, 81 (2) (1983) 304-311.

[13] R.A. Wilson, The finite simple groups, Springer-Verlag London Ltd, 2009.

[14] The GAP Group, GAP-Groups, Algorithms and programming, Vers.4.4.12 (2008). http://www. gap-system.org/ 